\title{
LEXICALL: Lexicon Construction for Foreign Language Tutoring
}

\author{
Bonnie J. Dorr \\ Department of Computer Science and \\ Institute for Advanced Computer Studies \\ University of Maryland \\ College Park, MD 20742, USA \\ bonnie@cs.umd.edu
}

\begin{abstract}
We focus on the problem of building large repositories of lexical conceptual structure (LCS) representations for verbs in multiple languages. One of the main results of this work is the definition of a relation between broad semantic classes and LCS meaning components. Our acquisition program-LEXICALL - takes, as input, the result of previous work on verb classification and thematic grid tagging, and outputs LCS representations for different languages. These representations have been ported into English, Arabic and Spanish lexicons, each containing approximately 9000 verbs. We are currently using these lexicons in an operational foreign language tutoring and machine translation.
\end{abstract}




\section{Introduction}

A wide range of new capabilities in NLP applications has been made possible by recent advances in lexical semantics (Carrier and Randall, 1993; Fillmore, 1968; Foley and Van Valin, 1984; Grimshaw, 1990; Gruber, 1965; Hale and Keyser, 1993; Jackendoff, 1983; Jackendoff, 1990; Jackendoff, 1996; Levin, 1993; Levin and Rappaport Hovav, To appear; Pesetsky, 1982; Pinker, 1989). Many of these researchers adopt the hypothesis that verbs can be grouped into broad classes, each of which corresponds to some combination of basic meaning components. This is the basic premise underlying our approach to multilingual lexicon construction. In particular, we have organized verbs into broad semantic classes and subsequently designed a language-independent representation, lexical conceptual structure (LCS), for each class; these representations have been ported into Arabic and Spanish on a per-class basis. The result is a large multi-lingual repository of verb entries: our Arabic and Spanish lexicons contain approximately 40,000 Arabic and Spanish entries each, of which 9,000 (per language) are verbs.

We are currently using these lexicons in an operational foreign language tutoring (FLT) system called Military Language Tutor (MILT). This system provides a wide range of lessons for use in language training. Because of the multilingual nature of the system (i.e., broad coverage of Arabic and Spanish), the representations we use must necessarily have crosslinguistic validity as well as coverage for diverse constructions that are likely to occur in lessons.

One of the tutoring lessons, the MicroWorld Lesson (see Figure 1) requires the capability for the language-learner to state domain-specific actions in a variety of different ways. For example, the language-learner might command the agent (pictured at the left in the graphical interface) to take the following action: Walk to the table and pick up the document. The same action should be taken if the user says: Go to the table and remove document, Retrieve the document from the table, etc. The LCS representation provides the capability to execute various forms of the same command without hardcoding them as part of the graphical interface.

In another tutoring lesson, Question-Answering, the student is asked to answer questions about a foreign language text that they have read. Their answer is converted into an LCS which is matched against a prestored LCS corresponding to an answer typed in by a human instructor (henceforth, called the "author"). The prestored LCS is an idealized form of the answer to a question, which can take one of many forms. Suppose, for example, the question posed to the user is: Where did Jack put the book? (or Adonde puso Jack el libro? in Spanish). The author's answer, e.g., Jack put the book in the trash, has been stored as an LCS by the tutoring system. If the student types Jack threw the book in the trash or Jack moved the book from the table into the trash, the system is able to match against the prestored LCS and determine that all three of these responses are semantically appropriate. 
Figure 1: MicroWorld Lesson in MILT

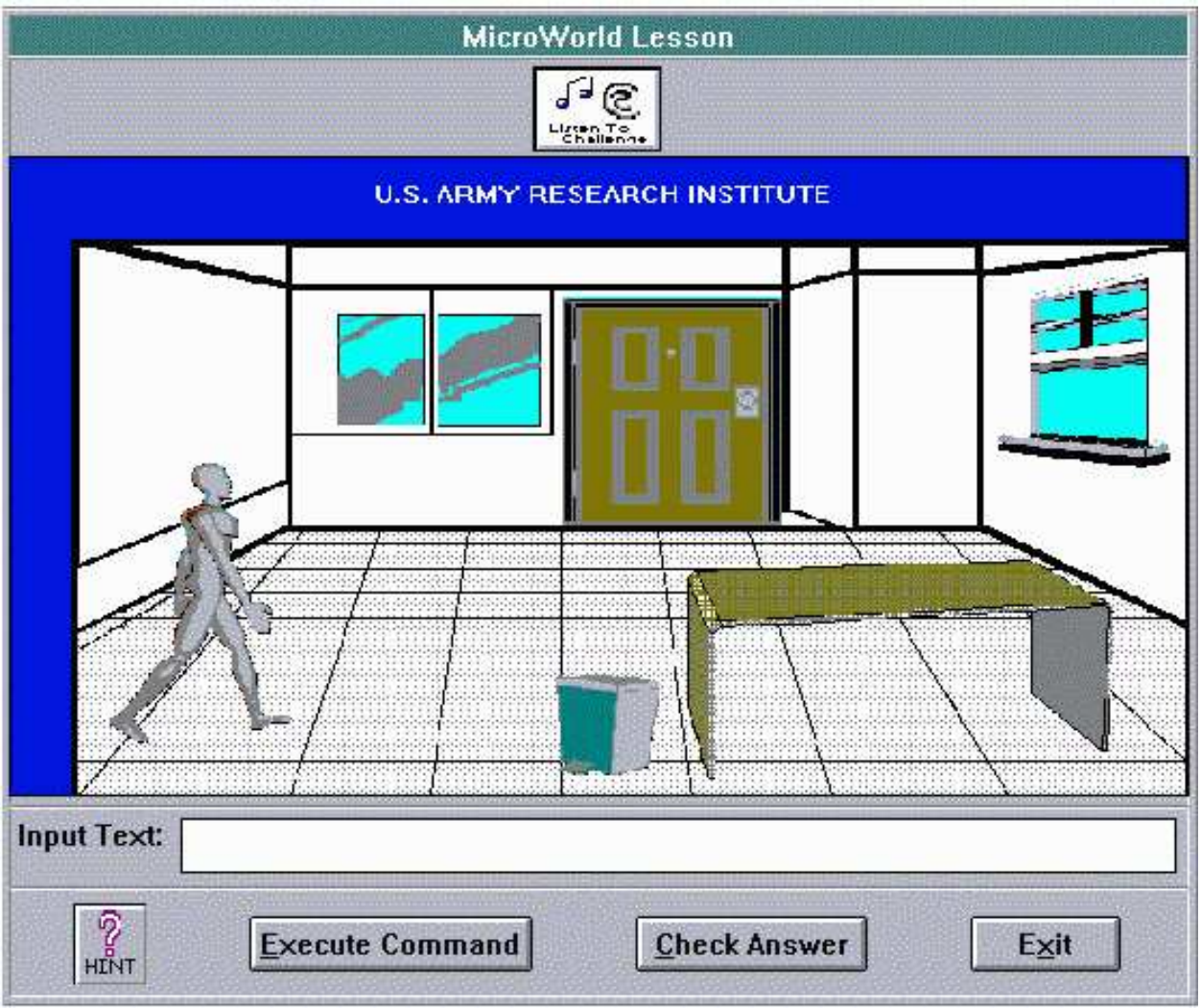


We have developed an acquisition program-LEXICALL - that allows us to construct LCS-based lexicons for the FLT system. LEXICALL is designed so that it can be used for multiple languages, and also for additional NLP applications (e.g., machine translation). One of the main results of this work is the definition of a relation between broad semantic classes (based on work by Levin (1993)) and LCS meaning components. The program builds on previous work, where verbs were classified automatically (Dorr and Jones, 1996; Dorr, To appear) and tagged with thematic grid information (Dorr et al., 1995). We use these pre-assigned classes and thematic grids as input to LEXICALL. The output is a set of LCS's corresponding to individual verb entries in our lexicon.

Previous research in automatic acquisition focuses primarily on the use of statistical techniques, such as bilingual alignment (Church and Hanks, 1990; Klavans and Tzoukermann, 1995; Wu and Xia, 1995), or extraction of syntactic constructions from online dictionaries and corpora (Brent, 1993). Others have taken a more knowledge-based (interlingual) approach (Lonsdale et al., 1995). Still others, e.g., Copestake et al. (1995), use English-based grammatical codes for acquisition of lexical representations.

Our approach differs from these in that it exploits certain linguistic constraints that govern the relation between a word's surface behavior and its corresponding semantic class. We demonstrate that a LCS representation can be assigned to each semantic class. These, in turn, are ported into multiple languages. We first show how the LCS is used in a FLT system. We then describe the techniques used for automatic acquisition of LCS-based lexicons.

\section{Application of the LCS Representation to FLT}

One of the types of knowledge that must be captured in FLT is linguistic knowledge at the level of the lexicon, which covers a wide range of information types such as verbal subcategorization for events (e.g., that a transitive verb such as hit occurs with an object noun phrase), featural information (e.g., that the direct object of a verb such as frighten is animate), thematic information (e.g., that Mary is the agent in Mary hit the ball), and lexical-semantic information (e.g., spatial verbs such as throw are conceptually distinct from verbs of possession such as give). By modularizing the lexicon, we treat each information type separately, thus allowing us to vary the degree of dependence on each level so that we can address the question of how much knowledge is necessary for the success of the particular NLP application.

This section describes the use of the LCS representation in a question-answering component of the MILT system (Sams, 1993; Weinberg et al., 1995). As described above, the LCS representation is used as the basis of matching routines for assessing students' answers to free response questions about a short foreign language passage. In order to inform the stu- 
dent whether a question has been answered correctly, the author of the lesson must provide the desired response in advance. The system parses and semantically analyzes the author's response into a corresponding LCS representation which is then prestored in a database of possible responses. Once the question answering lesson is activated, each of the student's responses is parsed and semantically analyzed into a LCS representation which is checked for a match against the corresponding prestored LCS representation. The student is then informed as to whether the question has been answered correctly depending on how closely the student's response LCS matches the author's prestored LCS.

Table 1: Correspondence Between NLP Output and Tutor Feedback

\begin{tabular}{|l|l|l|l|}
\hline \multicolumn{4}{|c|}{ System Prompt: Where did Jack put the book? } \\
\hline Student Answer & Prestored Answer & Matcher Output & Feedback \\
\hline Jack threw the book in the trash & Jack threw the book in the trash & exact match & "That's right" \\
\hline Jack put the book in the trash & Jack threw the book in the trash & missing MANNER & "How?" \\
\hline Jack threw the book in the trash & Jack put the book in the trash & extra MANNER & "You're assuming things" \\
\hline Jack is friendly & Jack put the book in the trash & mismatch primitive & "Please reread" \\
\hline Jack threw the book & Jack put the book in the trash & missing argument & "Where?" \\
\hline
\end{tabular}

Consider what happens in a lesson if the author has specified that a correct answer to the question Adónde puso Jack el libro? in Spanish is Jack tiró el libro a la basura ('Jack threw out the book into the trash'). This answer is processed by the system to produce the following LCS:

$$
\begin{aligned}
& \text { [Event CAUSE } \\
& \text { ([Thing JACK], } \\
& \text { [Event } \mathrm{GO}_{\text {Loc }} \\
& \text { ([Thing } \mathrm{BOOK}] \text {, } \\
& \text { [Fath } \mathrm{TO}_{\text {Loc }} \\
& \left.\left.\left.\left(\left[\left[_{\text {Fosition }} \operatorname{AT}_{\text {Loc }}\left(\left[_{\text {Thing }} \text { BOOK }\right],{ }_{\text {Thing }} \text { TRASH }\right]\right)\right]\right)\right]\right)\right] \text {, } \\
& \text { [Manner THROWINGLY])] }
\end{aligned}
$$

The LCS is stored by the tutor and then later matched against the student's answer. If the student types Jack movio' el libro de la mesa a la basura ('Jack moved the book from the table to the trash'), the system must determine if these two match. The student's sentence is processed and the following LCS structure is produced:

$$
\begin{aligned}
& {[\text { Event } \mathrm{CAUSE}} \\
& \left(\left[_{\text {Thing }} \mathrm{JACK}\right],\right.
\end{aligned}
$$




$$
\begin{aligned}
& \text { [Event } \mathrm{GO}_{\text {Loc }} \\
& \text { ( } \text { Thing }_{\text {ThOOK }} \text {, } \\
& \text { [Fath } \mathrm{TO}_{\text {Loc }}\left(\left[_{\text {Position }} \mathrm{AT}_{\text {Loc }}\right.\right. \\
& \left.\left.\left(\left[{ }_{\text {Thing }} \text { BOOK], [Thing TRASH] }\right)\right]\right)\right] \text {, } \\
& \text { [Fath } \text { FROM }_{\text {Loc }}\left(\left[_{\text {Fosition }} \text { AT }_{\text {Loc }}\right.\right. \\
& \left.\left.\left.\left.\left.\left.\left.\left.\left(\left[{ }_{\text {Thing }} \text { BOOK }\right] \text {, }{ }_{\text {Thing }} \text { TABLE }\right]\right)\right]\right)\right]\right)\right]\right)\right]
\end{aligned}
$$

The matcher compares these two, and produces the following output:

\section{Missing: MANNER THROWINGLY}

Extra: FROM LOC

The system identifies the student's response as a match with the prestored answer, but it also recognizes that there is one piece of missing information and one piece of extra information.

The "Missing" and "Extra" output is internal to the NLP component of the Tutor, i.e., this is not the final response displayed to the student. The system must convert this information into meaningful feedback so that the student knows how to repair the answer that was originally given. For example, the instructor can program the tutor to notify the student about the omitted information in the form of a 'How' question, or it can choose to ignore it. The extra information is generally ignored, although it is recorded in case the instructor decides to program the system to notify the student about this as well. The full range of feedback is not presented here. Some possibilities are summarized (in English) in Table 1 (adapted from (Holland, 1994)). Note that the main advantage of using the LCS is that it allows the author to type in an answer that is general enough to match any number of additional answers.

\section{Overview of LCS Acquisition}

Our work on construction of LCS lexicons has focused primarily on building entries for verbs. ${ }^{1}$ This section outlines our approach to verb acquisition.

LEXICALL requires three inputs for acquisition of verb entries: a semantic class, a thematic grid, and a lexeme, which we will henceforth abbreviate as "class/grid/lexeme". An example of input/output for the system is shown here:

(3) Acquisition of LCS for: touch

Input: 47.8 ; _th_loc; "touch"

Output:

(be loc (* thing 2) (at loc (thing 2) (* thing 11)) (touchingly 26))

\footnotetext{
${ }^{1}$ However, we have approximately 30,000 non-verb entries per language.
} 
The lexeme is, by convention, an English-like word whose morphological variant ultimately fills some position in the LCS definition. The verb that will be associated with the resulting LCS is not necessarily the same as this lexeme. For example, the Spanish counterpart of touch, also uses the "touch" lexeme. ${ }^{2}$

The semantic class label 47.8 above is taken from Levin's 1993 book (Verbs of Contiguous Location), i.e., the class to which the verb touch has been assigned. ${ }^{3}$ The thematic grid (_th_loc) is taken from a set of grid assignments previously assigned by (Dorr et al., 1995). The following conventions are adopted for thematic grid specifications: (1) Any thematic role preceded by an underscore (_) is obligatory; (2) Any thematic role preceded by a comma (,) is optional; (3) Any thematic role that is followed by a parenthesized preposition must necessarily be contained in a phrase headed by that preposition.

Table 2: Sample Templates Stored in the LCS Database

\begin{tabular}{|c|c|c|c|}
\hline Category & Verb & Class & LCS \\
\hline \multirow[t]{2}{*}{ Location } & suspend & 9.2 & {$\left[\mathrm{CAUSE}\left(\mathrm{X},\left[\mathrm{BE}_{\mathrm{Loc}}\left(\mathrm{Y},\left[\mathrm{AT}_{\mathrm{Loc}}(\mathrm{Y}, \mathrm{Z})\right]\right)\right],[\mathrm{BY}\langle\mathrm{MANNER}\rangle]\right)\right]$} \\
\hline & touch & 47.8 & {$\left[\mathrm{BE}_{\mathrm{Loc}}\left(\mathrm{Y},\left[\mathrm{AT}_{\mathrm{Loc}}(\mathrm{Y}, \mathrm{Z})\right],[\mathrm{BY}\langle\mathrm{MANNER}\rangle]\right)\right]$} \\
\hline \multirow[t]{2}{*}{ Motion } & abandon & 51.2 & {$\left[\mathrm{GO}_{\mathrm{Loc}}\left(\mathrm{Y},\left[\langle\operatorname{DIRECTION}\rangle_{\mathrm{Loc}}\left(\mathrm{Y},\left[\mathrm{AT}_{\mathrm{Loc}}(\mathrm{Y}, \mathrm{Z})\right]\right)\right]\right)\right]$} \\
\hline & float & 51.3 .1 & {$\left[\mathrm{GO}_{\mathrm{Loc}}(\mathrm{Y},[\mathrm{BY}\langle\mathrm{MANNER}\rangle])\right]$} \\
\hline \multirow[t]{2}{*}{ Placement } & adorn & 9.8 & $\begin{array}{l}\text { [CAUSE }(\mathrm{X}, \\
\text { [GO Ident }(\mathrm{Y}, \\
{\left[\text { TOWARD }_{\text {Ident }}(\mathrm{Y},\right.} \\
{\left[\mathrm{AT}_{\text {Ident }} \quad\left(\mathrm{Y}, \quad\left[\langle\text { STATE }\rangle_{\text {Ident }} \quad\left(\left[\langle\text { WITH }\rangle_{\text {Poss }}\right.\right.\right.\right.\right.} \\
\mathrm{Z})])])]]])])]\end{array}$ \\
\hline & spill & 9.5 & {$\left[\mathrm{CAUSE}\left(\mathrm{X},\left[\mathrm{GO}_{\mathrm{Loc}}(\mathrm{Y})\right],[\mathrm{BY}\langle\mathrm{MANNER}\rangle]\right)\right]$} \\
\hline
\end{tabular}

One piece of information that is not shown above is the mapping between the class/grid/lexeme input and the LCS representation. Using Levin's publicly available online index as a starting point, we have hand-constructed a database containing 191 LCS templates, i.e., one for verb class. These templates serve as the basis of instantiated output such as that of (3) above. A full entry in the database includes a list of possible lexemes, a thematic grid, and a LCS template:

(4) Class 47.8: adjoin, intersect, meet, touch, ...

\footnotetext{
${ }^{2}$ This is not to say that the generated LCS's for all translation equivalents are identical. Shortly, we will see a case where the LCS's produced by the program for two translation equivalents are annotated differently.

${ }^{3}$ Verbs not occurring in Levin's book are also assigned to classes using techniques described in (Dorr and Jones, 1996; Dorr, To appear).
} 


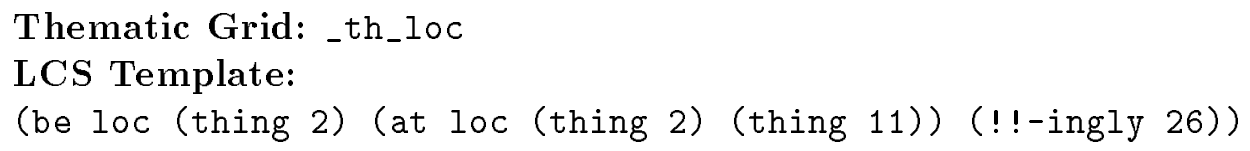

The semantic class and lexeme, together, uniquely identify the word sense, or LCS template, to which the verb refers. ${ }^{4}$

Note that the !! acts as a wildcard; it will be filled by the lexeme itself, and the resulting form is called a constant, i.e., the idiosyncratic part of the meaning that distinguishes among members of a verb class (in the spirit of (Grimshaw, 1993; Levin and Rappaport Hovav, To appear; Pinker, 1989; Talmy, 1985)). In addition, the output annotations (such as *) shown in (3) do not appear in the LCS template in (4). This is where the language-specific information is abstracted away from the language-independent meaning components. It is the instantiated lexical entry for touch that will have this type of information.

It is important to point out that, while Levin's classification provides a unique and extensive catalog of verb classes (such as change of state), it does not define the underlying meaning components of each class. One of the main contributions of our work is that it provides a relation between Levin's classes and meaning components as defined in the LCS representation. For example, we are able to determine whether the verb has an Identificational component of meaning in the LCS simply by checking whether the verb is in the Change of State class. The verbs break and cut have this component of meaning in the LCS representation, whereas, hit and touch do not. Table 2 shows three broad semantic categories and example verbs along with their associated LCS representations. ${ }^{5}$

In addition to defining a mapping between semantic classes and LCS meaning components, we have generated LCS templates for 26 additional classes that are not included in Levin's system. Several of these correspond to verbs that take sentential complements (such as coerce). The additional classes include, among others: Coerce Verbs (blackmail, bribe, cajole, ...); Conspire Verbs (legislate, protest, rebel, ...); Exceed Verbs (exceed, excel, outbid, ...); Penetrate Verbs (penetrate, permeate, pervade, ...).

\section{Language-Specific Annotations}

As described above, language-specific information such as the *-marker is not included in the templates of the LCS database. This information is added to the templates by processing the components of thematic grid specifications. In our on-going example (3), the thematic grid is _th_loc. From this it is determined that the theme and the location

\footnotetext{
${ }^{4}$ The words are assumed to have been disambiguated by techniques described in (Dorr and Jones, 1996; Dorr, To appear), during the assignment to semantic classes.

${ }^{5} \mathrm{LCS}$ constituents that contain angle-bracketed "constants" correspond to the !! wildcard. For example, [BY $\langle$ MANNER $)]$ corresponds to ! !-ingly.
} 
are both obligatory (in English) and should be annotated as such in the instantiated LCS. This language-specific correspondence is indicated through the use of the *-marker, which specifies the positions that are filled in by other LCS entries during sentence processing.

To illustrate this point, consider the structural divergence between the following English/Spanish equivalents; the lexical entries for the main verbs are included for comparison:

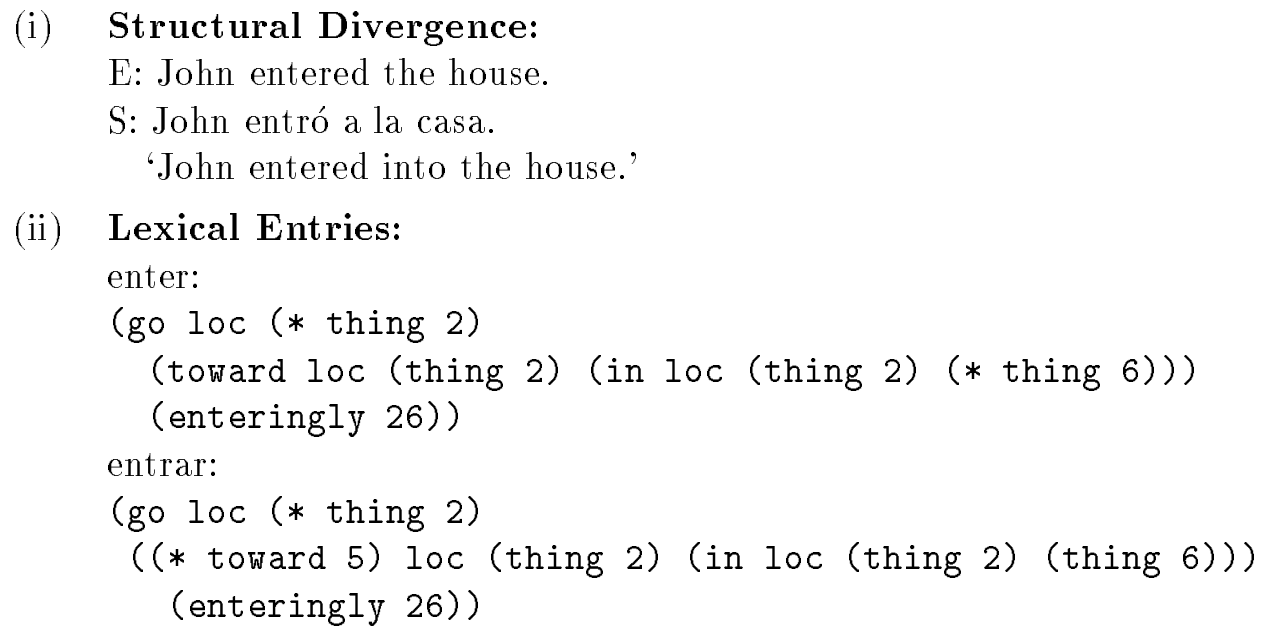

The English sentence differs structurally from the Spanish in that the noun phrase the house corresponds to a prepositional phrase a la casa. The lexicon entries for enter and entrar both mean "X (= Thing 2) goes into location $\mathrm{Y}$ (= Thing 6)," but the distinction shown in (5)(i) is characterized by different positionings of the $*$-marker in (5)(ii). In the LCS entries, variable positions (designated by numbers, such as 2 and 6 ) are used in place of the ultimate fillers such as JOHN and HOUSE. A *-marked leaf node must be filled in at the leaf level and a *-marked non-leaf node must be filled in through unification at the internal node indicated by the *-marker. Hence, in the above example the LCS for enter guarantees that the second argument (position 6) is filled in at the leaf node, whereas the LCS for entrar requires this same argument to be filled through unification at the (to loc ...) node (position 5). In this latter case, the Spanish preposition a (i.e., 'to/into') fulfills this requirement since it has an LCS that matches at that level:

(toward loc (thing 2) (in loc (thing 2) (thing 6))).

In addition to the $*$-marker, lexical entries contain other language-specific information: a : collocations slot for idiosyncratic prepositions and a : var_spec slot for information about variable positions (e.g., optionality of arguments). For brevity, we focus only on aspects of the acquisition process that affect the instantiation of the LCS template. The next section describes the construction of lexical entries in more detail. 
Table 3: Thematic Roles Corresponding to Numbered Positions in the LCS: Modifiers

\begin{tabular}{|c|c|}
\hline \multicolumn{2}{|r|}{ Arguments } \\
\hline No./Role & Description \\
\hline $1 / \mathrm{ag}$ & $\begin{array}{l}\text { Agent of CAUSE, LET, and } \\
\text { CAUSE_EXCHANGE }\end{array}$ \\
\hline $2 /$ th & $\begin{array}{l}\text { Theme of } \mathrm{GO}_{\text {Loc }}, \mathrm{GO}_{\text {Poss }}, \\
\mathrm{GO}_{\text {Ident }}, \mathrm{GO}_{\text {Circ }}, \mathrm{BE}_{\text {Poss }}, \\
\mathrm{BE}_{\text {Ident }}, \mathrm{BE}_{\text {Circ }}\end{array}$ \\
\hline $2 / \exp$ & $\begin{array}{l}\text { Experiencer of } \mathrm{GO}_{\text {Perc }} \text { and } \\
\mathrm{BE}_{\text {Perc }}\end{array}$ \\
\hline $3 / \operatorname{src}()$ & $\begin{array}{l}\text { Path (from/from_away) be- } \\
\text { fore source }\end{array}$ \\
\hline $4 / \mathrm{src}$ & Source of Loc, Ident, Poss \\
\hline $5 / \operatorname{goal}()$ & $\begin{array}{l}\text { Path (to/toward) preceding } \\
\text { goal }\end{array}$ \\
\hline $6 /$ goal & Goal of Loc, Ident, Poss \\
\hline $7 / \operatorname{perc}()$ & $\begin{array}{l}\text { Perceived particle preceding } \\
\text { perceived item }\end{array}$ \\
\hline $8 /$ perc & Perceived item \\
\hline $9 /$ pred & Identificational predicate \\
\hline $10 / \operatorname{loc}()$ & $\begin{array}{l}\text { Locational particle preceding } \\
\text { locative goal }\end{array}$ \\
\hline $11 / \mathrm{loc}$ & Locational predicate \\
\hline $12 /$ poss & Possessional predicate \\
\hline $13 /$ time & $\begin{array}{l}\text { Temporal particle preceding } \\
\text { time }\end{array}$ \\
\hline 14/time() & Time for Temp field \\
\hline
\end{tabular}

\begin{tabular}{|l|l|}
\hline \multicolumn{2}{|c|}{ Modifiers } \\
\hline No./Role & Description \\
\hline $\begin{array}{l}15 / \text { mod- } \\
\text { oss() }\end{array}$ & $\begin{array}{l}\text { Possessional particle preced- } \\
\text { ing possessed item }\end{array}$ \\
\hline $\begin{array}{l}16 / \text { mod- } \\
\text { poss }\end{array}$ & Possessed item modifier \\
\hline $17 /$ ben() & $\begin{array}{l}\text { Intentional particle preceding } \\
\text { benefactive modifier }\end{array}$ \\
\hline $18 /$ ben & Benefactive modifier \\
\hline $19 /$ instr() & $\begin{array}{l}\text { Instrumental particle preced- } \\
\text { ing instrument modifier }\end{array}$ \\
\hline $20 /$ instr & Instrument modifier \\
\hline $21 /$ purp() & $\begin{array}{l}\text { Intentional particle preceding } \\
\text { purpose modifier }\end{array}$ \\
\hline $22 /$ purp & Purpose modifier \\
\hline $\begin{array}{l}23 / \text { mod- } \\
\text { loc() }\end{array}$ & $\begin{array}{l}\text { Locational particle preceding } \\
\text { location modifier }\end{array}$ \\
\hline $24 /$ mod-loc & Location modifier \\
\hline $25 /$ manner() & Manner component \\
\hline $26 / ! !-i n g l y$ & Conflated manner component \\
\hline $27 /$ prop & Event or state \\
\hline $\begin{array}{l}28 / \text { mod- } \\
\text { prop }\end{array}$ & Event or state \\
\hline $\begin{array}{l}29 / \text { mod- } \\
\text { pred() }\end{array}$ & $\begin{array}{l}\text { Identificational particle pre- } \\
\text { ceding property modifier }\end{array}$ \\
\hline $\begin{array}{l}30 / \text { mod- } \\
\text { pred }\end{array}$ & Property modifier \\
\hline
\end{tabular}




\section{Construction of Lexical Entries}

As illustrated above, LCS templates use a Lisp-like representation. Consider Fill Verbs $(9.8):^{6}$

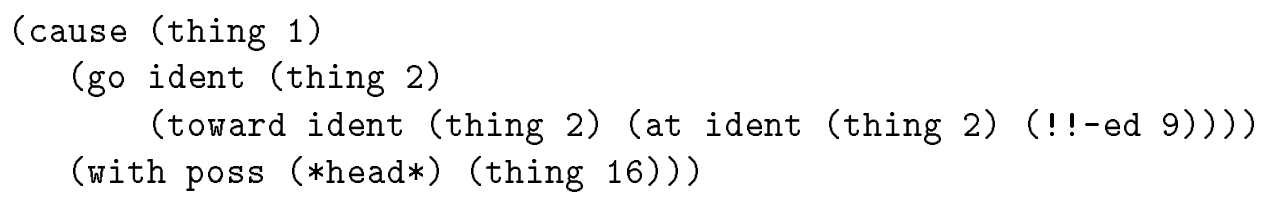

This list structure recursively associates logical heads with their arguments and modifiers. The logical head is represented as a primitive/field combination, e.g., $\mathrm{GO}_{\text {Loc }}$ is represented as (go loc ...). The arguments for CAUSE are (thing 1) and (go ident ...). The substructure GO itself has two arguments (thing 2) and (toward ident ...). Modifiers are non-argument positions in the LCS, e.g., the (with poss ...) substructure in the representation above (as in the sentence She trimmed the tree with tinsel). ${ }^{7}$ Note that the ! !-ed constant refers to a resulting state, e.g., adorned for the verb adorn.

The numbers in the representation are codes that map between LCS positions and their corresponding thematic roles. In the LCS framework, thematic roles provide semantic information about properties of the argument and modifier structures. We adopt the thematic relations hypothesis (Fillmore, 1968; Gruber, 1965) which states that the semantics of location and motion can be generalized to additional semantic fields.

Thematic roles have long been the focus of conceptual structure studies. In the work of (Jackendoff, 1983), thematic roles are associated with the notion of fields (Spatial, Temporal, Possessional, Circumstantial, and Existential) and have continued their prominence in more recent theories of lexical semantics (Dowty, 1991; Jackendoff, 1990). Subsequently, additional thematic roles have been proposed for new semantic fields (Dorr et al., 1993): Perceptual (for Events and States), Intentional (for modifiers such as purpose clauses), and Instrumental (for instrument modifiers).

Tables 3 shows the relation of thematic roles to components of the LCS representation for arguments and modifiers. These numbers enter into the construction of LCS entries: they correspond to argument positions in the LCS template (extracted using the class/grid/lexeme specification). Information is filled into the LCS template using these numbers, coupled with the thematic grid tag for the particular word being defined.

\footnotetext{
${ }^{6}$ Some of the 9.8 verbs are: adorn, anoint, bandage, bathe, bestrew, bind, blanket, block, blot, festoon, fill, fleck, flood, frame, garland, garnish, imbue, mottle, ornament, pad, pave, plate, plug, pollute, replenish, stud, suffuse, surround, swaddle, swathe, taint, tile, trim, veil.

${ }^{7}$ The $*$ head* symbol is a place-holder that points to the root (cause) of the overall lexical entry. Modifiers, such as instrumental phrases, typically include this symbol.
} 


\subsection{Fundamentals}

LEXICALL is designed to locate the appropriate LCS template (out of the 191 handconstructed database entries) using the class/grid pairing as an index, and to then determine the language-specific annotations to instantiate for that template. Although it is desirable to generate the most representative LCS definition for a lexical item, this is not always possible. A verb class usually contains template variations for the verbs they cover, i.e., subclasses. These are relatively general cases - verbs in a class show similar properties, but the class is further refined by certain template variations.

Thus, for each verb to be defined, LEXICALL uses the class/grid pairing to extract the smallest possible set of LCS templates that comply with the class/grid pairing. It is also possible to index into the database by the grid only. The semantic class is considered an optional argument to the acquisition procedure, and generally more than one LCS definition will be returned in such cases. The grid information cannot be omitted, however.

Once a set of LCS templates is selected, the next step is to place *-markers in the appropriate positions. To do this, the program must take into account structural divergence possibilities (e.g., the case given in example (5)(i) above). The default position of the *marker is the left-most occurrence of the LCS node corresponding to a particular thematic role. However, if a preposition occurs in the grid, the *-marker may be placed differently. In such a case, a primitive representation (e.g., (to loc (at loc)) ) is extracted using a set of predefined mappings (to be described in Section 5.2, case III). If this representation corresponds to a subcomponent of the LCS template, the program recognizes this as a match against the grid, and the *-marker is placed in the template at the level where this match occurs (as in the entry for entrar given in (5)(ii) above).

If a preposition occurs in the grid but there is no matching primitive representation, the preposition is considered to be a collocation, and it is placed in the collocations slot of the lexical entry - which indicates that the LCS already covers the semantics of the verb and the preposition is an idiosyncratic variation (as in learn about, know of, etc.).

If a preposition is required but it is not specified (i.e., empty parentheses ()), then the *-marker is positioned at the level dominating the node that corresponds to that rolewhich indicates that several different prepositions might apply (as in put on, put under, put through, etc.).

\subsection{Processing thematic grids}

The input to LEXICALL is a class/grid/lexeme specification, where each piece of information is separated by a hash sign $(\#)$ :

$\langle$ class $\rangle \#\langle$ grid $\rangle \#<$ lexeme $\rangle \#<$ other semantic information $>$ For example, the input specification for the verb replant (a word not classified by Levin) is: 


\section{7\#_ag_th,mod-poss (with)\#replant\#!!-ed = planted (manner $=$ again)}

This input indicates that the class assigned to replant is 9.7 (Levin's Spray/Load verbs) and its grid has an obligatory agent (ag), theme (th), and an optional possessional modifier with preposition with (mod-poss(with)). By default, arguments are obligatory and modifiers are optional. If a role in the thematic grid overrides this default, the information is recorded in the var_spec slot of the lexical item.

The information following the final \# is optional; this information was previously handadded to the assigned thematic grids. In the current example, the !!-ed designates the form of the constant planted which, in this case, is a morphological variant of the lexeme replant. ${ }^{8}$ Also, the manner again is specified as an additional semantic component.

Returning to our example (5) above, the verbs entrar and entrar have the following class/grid/lexeme specifications:

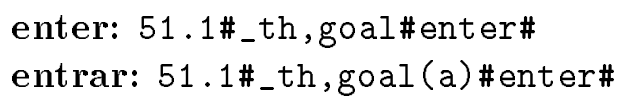

LEXICALL produces the English and Spanish LCS output shown in (5)(ii) for these two specifications.

For presentational purposes, the remainder of this section uses English examples. However, as the above examples illustrate, the class/grid/lexeme specification carries over to other languages as well. In fact, we have used the same acquisition program, without modification, for building our Spanish and Arabic LCS-based lexicons, each of size comparable to our English LCS-based lexicon.

There are a number of different cases for which the class/grid/lexeme specification needs to be processed for the construction of lexical entries. We will look at four cases in detail.

\section{Thematic Roles without Prepositions}

(8) Example: The flower decorated the room.

Input: $9.8 \#$ mod-poss_th\#decorate\#

Template: (be ident (thing 2) (at ident (thing 2)

$(! !$-ed 9))

(with poss (*head*) (thing 16)))

\footnotetext{
${ }^{8}$ The constant takes one of several forms, including: ! !-ingly for a manner, ! !-er for an instrument, and ! !-ed for resulting states. If this information has not been hand-added to the class/grid/lexeme specification (as is the case with most of the verbs), a default morphological process produces the appropriate form from the lexeme. Note that, in some cases, the morphological process might produce unexpected surface forms (e.g., hoer instead of hoe). Because we take the constant to be a label for semantic meaning-having a different status from other components of meaning (e.g., GO, BE, etc.) - the precise form of the constant is inconsequential; thus, a close approximation is sufficient for our purposes.
} 
This case involves thematic roles that do not require prepositions for any of the argument or modifier variables. Two thematic roles, th and mod-poss, are specified for the above sense of the English verb decorate. Table 3 indicates that the thematic code numbers are 2 and 16, respectively. The following output shows the result of positioning the $*$-markers and the constant decorated in the LCS template:

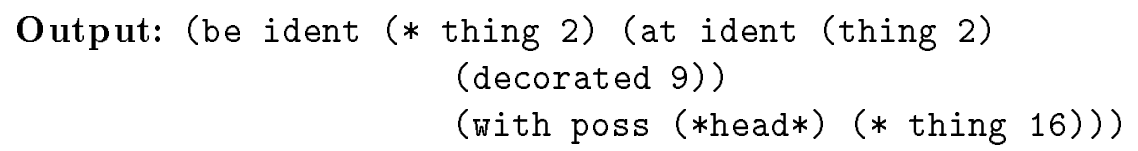

Duplicate occurrences of a node are taken to be coindexed; thus, only one such node needs to be $*$-marked. Our convention is to mark the left-most occurrence (e.g., the first (thing 2) node above).

\section{Thematic Roles with Unspecified Prepositions}

(10) Example: We parked the car near the store.

We parked the car in the garage.

Input: 9.1\#_ag_th_goal()\#park\#

Template: (cause (thing 1)

(go loc (thing 2) (toward loc (thing 2)

([at] loc (thing 2) (thing 6))) )

(! !-ingly 26))

The input for this example indicates that there is necessarily a goal headed by a preposition, although the preposition is not given explicitly. The thematic roles are ag, th, and goal() with the corresponding code numbers 1,2 , and 6 , as given in Table 3 . The resulting output is the following:

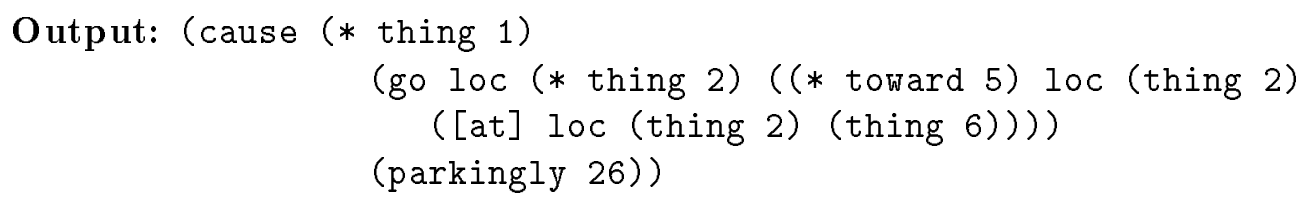

The variable positions for ag and th are marked just as in the previous case, whereas goal() requires a different treatment. When there is no preposition specified for the thematic roles with required prepositions, the *-marker is associated with a LCS node dominating the position corresponding to the relevant thematic role (i.e., (toward ...) in the example above). The ability to underspecify the preposition allows for a number of surface-sentence variants such as park in, park on, park under, etc. (In such cases, the bracketed primitive [at] node will be converted into an unbracketed positional primitive-e.g., in, on, or under-during analysis of the surface sentence.) 


\section{Thematic roles with Specified Prepositions}

(12) Example: We decorated the room with flowers.

Input: 9.8\#_ag_th,mod-poss(with)\#decorate\#

Template: (cause (thing 1)

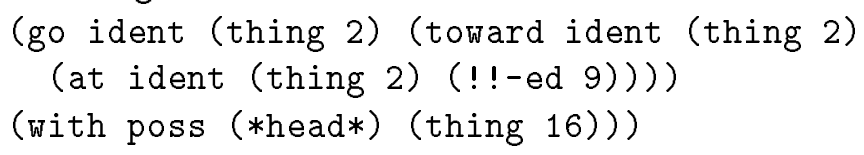

Specified prepositions can occur both for argument and modifier thematic roles. This example demonstrates the process for a modifier role, however the same also applies to the argument roles. Here, the mod-poss role requires the preposition with in the modifier position. Thus, the output of LEXICALL is:

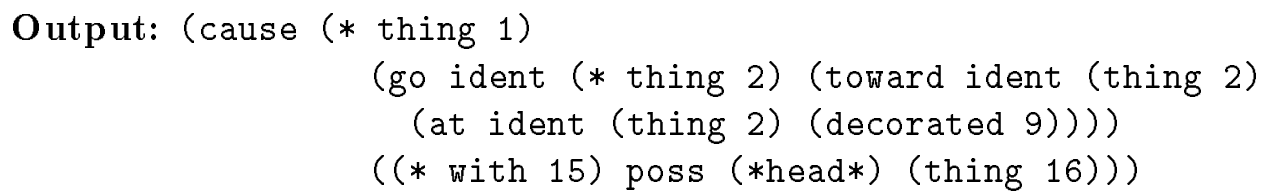

In order to determine the position of the *-marker for a thematic role with a required preposition, LEXICALL consults a set of predefined mappings between prepositions (or postpositions, in a language like Korean) and their corresponding primitive representations. ${ }^{9}$ In the current case, the preposition with is mapped to the following primitive representation: (with poss). Since this matches a sub-component of the LCS template, the program recognizes this as a match against the grid, and the $*$-marker is placed in the template at the level of with.

\section{Thematic Roles with Extra information}

(14) Example: They afforested the farm.

Input: 9.8\#_ag_goal\#afforest\#!!-ingly = afforestingly ( $t h=$ forest)

Template: (cause (thing 1)

(go loc (thing 2) (toward loc (thing 2)

(at loc (thing 2) (thing 6))) )

(with loc (*head*) (thing 16)))

(! !-ingly 26))

\footnotetext{
${ }^{9}$ We have defined approximately 100 such mappings per language. For example, the mapping produces the following primitive representations for the English word to: (to loc (at loc)), (to poss (at poss)), (to temp (at temp)), (toward loc (at loc)), (toward poss (at poss)). We have similar mappings defined in Spanish and Arabic. For example, the following primitive representations are produced for the Spanish word $a$ : (at loc), (to loc (at loc)), (to poss (at poss)), (toward loc (at loc)).
} 
Recall that the input potentially has hand-coded information following the final \#. In such cases, the specification includes a constant (as in the manner afforestingly above) and sometimes extra information about LCS arguments. In the example above, the specification indicates that the verb afforest has an implicit theme in its meaning, i.e., forest. Thus, instead of annotating the theme (designated by the code 2 ) with a *-marker, this position is directly replaced by the primitive forest:

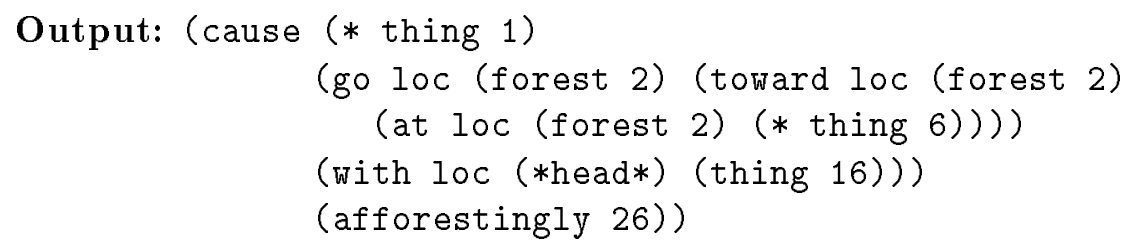

\section{Limitations and Conclusions}

We have described techniques for automatic construction of dictionaries for use in large-scale FLT. The dictionaries are based on a language-independent representation called lexical conceptual structure (LCS). Significant enhancements to LCS-based tutoring could be achieved by combining this representation with a mechanism for handling issues related to discourse and pragmatics. For example, although the LCS processor is capable of determining that the phrase in the trash partially matches the answer to Where did John put the book?, a pragmatic component would be required to determine that this answer is (perhaps) more appropriate than the full answer, He put the book in the trash. Representing conversational context and dynamic context updating (Traum et al., 1996; Haller, 1996; DiEugenio and Webber, 1996) would provide a framework for this type of response "relaxation." Along these same lines, a pragmatic component could provide a mechanism for determining that certain fully matched responses (e.g., John hurled the book into the trash) are not as "realistic sounding" as partially matched alternatives.

Initially, LEXICALL was designed to support the development of LCS's for English only; however, the same techniques can be used for multilingual acquisition. As the lexicon coverage for other languages expands, it is expected that our acquisition techniques will help further in the cross-linguistic investigation of the relationship between Levin's verb classes and the basic meaning components in the LCS representation. In addition, it is expected that verbs in the same Levin class may have finer distinctions than what we have specified in the current LCS templates.

We view the importation of LCS's from the English LCS database into Arabic and Spanish as a first approximation to the development of complete lexicons for these languages. The results have been hand-checked by native speakers using the class/grid/lexeme format (which is much easier to check than the fully expanded LCS's). The lexical verification 
process took only two weeks by the native speakers. We estimate that it would take at least 6 months to build such a lexicon from scratch (by human recall and data entry alone), and in such a case, the potential for error would be at least twice as high.

One important benefit of using the Levin classification as the basis of our program is that, once the mapping between verb classes and LCS representations has been established, we can acquire the LCS representation for a new verb (i.e., one not in Levin) simply by associating it with one of the 191 classes. We see our approach as a first step toward compression of lexical entries in that it allows lexicons to be stored in terms of the more condensed class/grid/lexeme specifications; these can expanded online, as needed, during sentence processing in the NLP application.

We conclude that, while human intervention is necessary for the acquisition of class/grid information, this intervention is virtually eliminated from the LCS construction process because of our provision of a mapping between semantic classes and primitive meaning components.

\section{Acknowledgements}

I would like to thank Jungshin Park and Mine Ulku Sencan for their aid in the development of certain components of the LEXICALL program. This work was supported, in part, by Army Research Office contract DAAL03-91-C-0034 through Battelle Corporation, NSF NYI IRI-9357731 and Logos Corporation, NSF CNRS INT-9314583, Advanced Research Projects Agency and ONR contract N00014-92-J-1929, Alfred P. Sloan Research Fellow Award BR3336, Army Research Institute contract MDA-903-92-R-0035 and Microelectronics and Design, Inc., and the University of Maryland General Research Board.

\section{References}

Michael Brent. 1993. Unsupervised Learning of Lexical Syntax. Computational Linguistics, $19: 243-262$.

Jill Carrier and Janet H. Randall. 1993. Lexical mapping. In Eric Reuland and Werner Abraham, editors, Knowledge and Language II: Lexical and Conceptual Structure, pages 119-142. Kluwer, Dordrecht.

Kenneth Church and P. Hanks. 1990. Word Association Norms, Mutual Information and Lexicography. Computational Linguistics, 16:22-29. 
Ann Copestake, Ted Briscoe, P. Vossen, A. Ageno, I. Castellon, F. Ribas, G. Rigau, H. Rodríguez, and A. Samiotou. 1995. Acquisition of Lexical Translation Relations from MRDS. Machine Translation, 9:183-219.

Barbara DiEugenio and Bonnie Lynn Webber. 1996. Pragmatic Overloading in Natural Language Instructions. International Journal of Expert Systems, 9(1):53-84.

Bonnie J. Dorr and Douglas Jones. 1996. Role of Word Sense Disambiguation in Lexical Acquisition: Predicting Semantics from Syntactic Cues. In Proceedings of the International Conference on Computational Linguistics, pages 322-333, Copenhagen, Denmark.

Bonnie J. Dorr, James Hendler, Scott Blanksteen, and Barrie Migdalof. 1993. Use of Lexical Conceptual Structure for Intelligent Tutoring. Technical Report UMIACS TR 93-108, CS TR 3161, University of Maryland.

Bonnie J. Dorr, Joseph Garman, and Amy Weinberg. 1995. From Syntactic Encodings to Thematic Roles: Building Lexical Entries for Interlingual MT. Machine Translation, $9: 71-100$.

Bonnie J. Dorr. To appear. Large-Scale Dictionary Construction for Foreign Language Tutoring and Interlingual Machine Translation. Machine Translation, 12(1).

David Dowty. 1991. The Effects of Aspectual Class on the Temporal Structure of Discourse: Semantics or Pragmatics? Language, 67:547-619.

Charles Fillmore. 1968. The Case for Case. In E. Bach and R. Harms, editors, Universals in Linguistic Theory, pages 1-88. Holt, Rinehart, and Winston.

William A. Foley and Robert D. Van Valin. 1984. Functional Syntax and Universal Grammar. Cambridge University Press, Cambridge.

Jane Grimshaw. 1990. Argument Structure. MIT Press, Cambridge, MA.

Jane Grimshaw. 1993. Semantic Structure and Semantic Content in Lexical Representation. unpublished ms., Rutgers University, New Brunswick, NJ.

Jeffrey S. Gruber. 1965. Studies in Lexical Relations. Ph.D. thesis, MIT, Cambridge, MA.

Ken Hale and Samuel J. Keyser. 1993. On Argument Structure and Lexical Expression of Syntactic Relations. In Ken Hale and Samuel J. Keyser, editors, The View from Building 20: Essays in Honor of Sylvain Bromberger. MIT Press, Cambridge, MA.

Susan Haller. 1996. Planning Text About Plans Interactively. International Journal of Expert Systems, 9(1):85-112. 
Melissa Holland. 1994. Intelligent Tutors for Foreign Languages: How Parsers and Lexical Semantics can Help Learners and Assess Learning. In Proceedings of the Educational Testing Service Conference on Natural Language Processing Techniques and Technology in Assessment and Education, Princeton, NJ: ETS.

Ray Jackendoff. 1983. Semantics and Cognition. MIT Press, Cambridge, MA.

Ray Jackendoff. 1990. Semantic Structures. MIT Press, Cambridge, MA.

Ray Jackendoff. 1996. The Proper Treatment of Measuring Out, Telicity, and Perhaps Even Quantification in English. Natural Language and Linguistic Theory, 14:305-354.

Judith L. Klavans and Evelynne Tzoukermann. 1995. Dictionaries and Corpora: Combining Corpus and Machine-Readable Dictionary Data for Building Bilingual Lexicons. Machine Translation, 10:185-218.

Beth Levin and Malka Rappaport Hovav. To appear. Building Verb Meanings. In M. Butt and W. Gauder, editors, The Projection of Arguments: Lexical and Syntactic Constraints. CSLI.

Beth Levin. 1993. English Verb Classes and Alternations: A Preliminary Investigation. Chicago, IL.

Deryle Lonsdale, Teruko Mitamura, and Eric Nyberg. 1995. Acquisition of Large Lexicons for Practical Knowledge-Based MT. Machine Translation, 9:251-283.

David Pesetsky. 1982. Paths and Categories. Ph.D. thesis, MIT, Cambridge, MA.

Steven Pinker. 1989. Learnability and Cognition: The Acquisition of Argument Structure. MIT Press, Cambridge, MA.

Michelle Sams. 1993. An Intelligent Foreign Language Tutor Incorporating Natural Language Processing. In Proceedings of Conference on Intelligent Computer-Aided Training and Virtual Environment Technology, NASA: Houston, TX.

Leonard Talmy. 1985. Lexicalization Patterns: Semantic Structure in Lexical Forms. In T. Shopen, editor, Language Typology and Syntactic Description 3: Grammatical Categories and the Lexicon, pages 57-149. University Press, Cambridge, England.

David R. Traum, Lenhart K. Schubert, Nathaniel G. Martin, Chung Hee Hwang, Peter Heeman, George Ferguson, James Allen, Massimo Poesio, and Marc Light. 1996. Knowledge Representation in the TRAINS-93 Conversation System. International Journal of Expert Systems, 9(1):173-223. 
Amy Weinberg, Joseph Garman, Jeffery Martin, and Paola Merlo. 1995. Principle-Based Parser for Foreign Language Training in German and Arabic. In Melissa Holland, Jonathan Kaplan, and Michelle Sams, editors, Intelligent Language Tutors: Theory Shaping Technology. Lawrence Erlbaum Associates, Hillsdale, NJ.

D. Wu and X. Xia. 1995. Large-Scale Automatic Extraction of an English-Chinese Translation Lexicon. Machine Translation, 9:285-313. 